Cite this: Soft Matter, 2014, 10, 1295

\title{
Manipulation of micro- and nanostructure motion with magnetic fields
}

Received 30th August 2013

Accepted 4th October 2013

DOI: $10.1039 / c 3 s m 52294 f$

www.rsc.org/softmatter

\author{
Roger S. M. Rikken, ${ }^{\text {ab }}$ Roeland J. M. Nolte, ${ }^{b}$ Jan C. Maan, ${ }^{a}$ Jan C. M. van Hest, ${ }^{b}$ \\ Daniela A. Wilson ${ }^{\mathrm{b}}$ and Peter C. M. Christianen ${ }^{\star a}$
}

In this review we will focus on how magnetic fields can be used to manipulate the motion of various microand nanostructures in solution. We will distinguish between ferromagnetic, paramagnetic and diamagnetic materials. Furthermore, the use of various kinds of magnetic fields, such as homogeneous, inhomogeneous and rotating magnetic fields, is discussed. To date most research has focused on the use of ferro- and paramagnetic materials, but here we also describe the possibilities of magnetic manipulation of diamagnetic materials. Since the vast majority of soft matter is diamagnetic, this paves the way for many new applications to manipulate the motion of micro- and nanostructures.

\section{Introduction}

Controlled motion of micro- and nanostructures in solution is a key ingredient for transport and assembly of nanomaterials in various applications. For instance, in drug delivery it is important that carriers can be accurately manoeuvred to pick up drugs at one location and release it at another. ${ }^{1}$ Another example is the separation of blood cells, bacteria or cancer cells from blood samples in microfluidics, which is crucial for diagnosing diseases or infections. ${ }^{2-5}$ Influencing motion and orientation of

${ }^{a}$ High Field Magnet Laboratory (HFML), Radboud University Nijmegen, Toernooiveld 7, 6525 ED Nijmegen, The Netherlands. E-mail: p.christianen@science.ru.nl ${ }^{b}$ Institute of Molecules and Materials, Radboud University Nijmegen, Heyendaalseweg 135, 6525 AJ, Nijmegen, The Netherlands. E-mail: d.wilson@science.ru.nl micro-objects also gives the ability to control their self-assembly in larger structures ${ }^{6}$ or induce nematic phases in suspensions of spheroidal particles. ${ }^{7}$

To master movement at the micro- and nanoscale, different concepts can be utilized, many of which are also found in nature. For instance, micro- and nanostructures can be moved along a predefined path. In cells this is done by kinesis proteins, which transport biomolecules by moving along tubulin fibers. ${ }^{\mathbf{8} 9}$ Another example is the conversion of rotational motion into linear translation. This concept is applied by flagella, the rotating tail that bacteria use for propulsion. ${ }^{8}$ Motion can also be induced by an external field gradient. For example, a proton gradient is necessary for inducing rotational movement in ATPsynthase, the protein responsible for ATP synthesis. ${ }^{8}$ Finally, steering of micro- and nanostructures can be achieved by

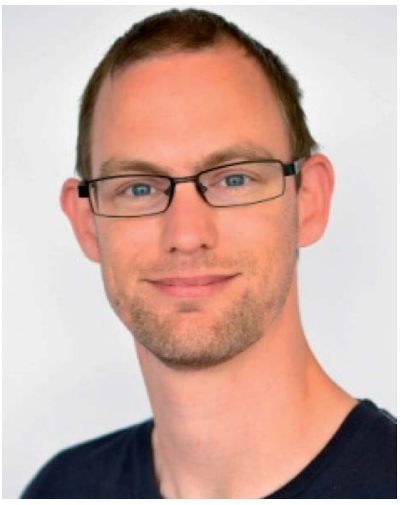

Roger S. M. Rikken received his BEd in Physics Teaching cum laude from the HAN University of Applied Sciences in 2007. He went on to study Natural Sciences at the Radboud University Nijmegen where he received his $B S c$ and $M S c$ cum laude in 2010 and 2012, respectively. He joined the Graduate School for Molecules and Materials in 2012, where he obtained a grant for a PhD position. He is currently doing his PhD at the High Field Magnet Laboratory and the Institute of Molecules and Materials departments of the Radboud University Nijmegen, where he is focusing on magnetic manipulation of self-assembled nanostructures.

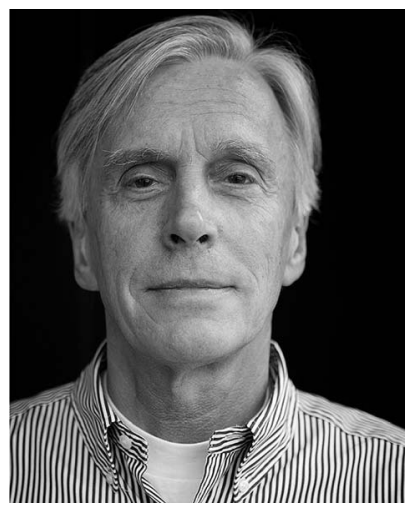

Roeland J. M. Nolte is an Emeritus Professor of Organic Chemistry at the Radboud University Nijmegen, The Netherlands, and a former Director of the Institute for Molecules and Materials of this university. He is a member of the Royal Netherlands Academy of Science and holds a special Royal Academy of Science Chair in Chemistry. His research interests span a broad range of topics at the interfaces of Supramolecular Chemistry, Macromolecular Chemistry, and Biomimetic Chemistry, in which he focuses on the design of catalysts and (macro)molecular materials. 
aligning them along an external vector field, such as electric and magnetic fields. This property is exploited by organisms that use the Earth's magnetic field for orientation, like certain birds, fish and magnetotactic bacteria. ${ }^{10}$ With such a diversity of examples, it is not surprising that many synthetic nanomotors are inspired by those found in nature.

Several review articles have been published over the years that discuss various nanomotors, for instance, molecular ${ }^{\mathbf{1 1}}$ and nanomotors with $^{\mathbf{1 2}}$ or without ${ }^{\mathbf{1 3}}$ moving parts. The motion of these nanomotors was induced by various stimuli such as electromagnetic radiation, ultrasound, chemical reactions and electric or magnetic fields. ${ }^{\mathbf{1 4 - 1 6}}$ In this review we will discuss propulsion and steering of micro- and nanostructures induced by external magnetic fields. Using magnetic fields for manipulation has several advantages. First of all, the response can be varied by

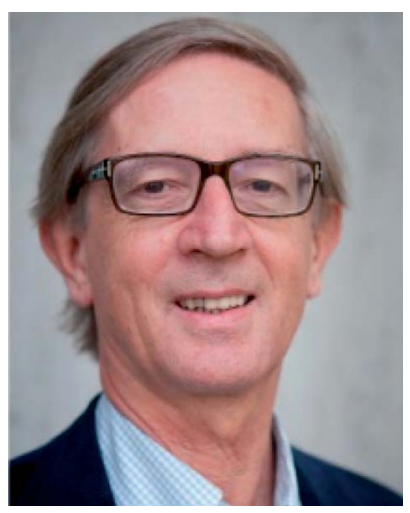

J. C. Maan (Jan Kees) is a full professor in experimental solid state physics with emphasis on the study of the properties of either top-down (semiconductor wells, dots and wires) nanostructures or bottom-up (through self-assembly). He is also the Director of the High Field Magnet Laboratory (HFML) which specializes in generating the highest possible $D C$ fields and exploiting them for research. He tries to combine these two activities by stimulating the creative use of high magnetic field in the field of nanostructures. Maan is the author of over 400 publications in the field of interest and is a knight in the Order of the Dutch Lion.

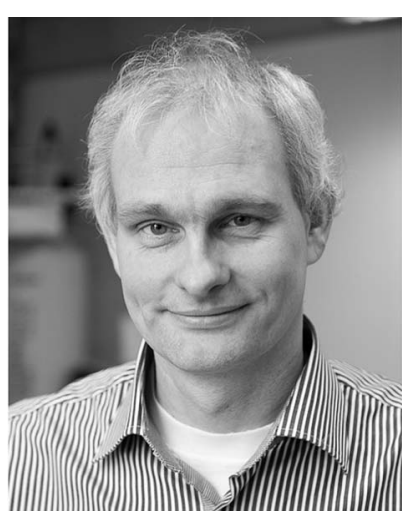

Jan C. M. van Hest obtained his PhD degree in 1996 at the Eindhoven University of Technology under the guidance of Prof. Bert Meijer. As a postdoctoral researcher he investigated the incorporation of noncanonical amino acids via protein engineering under the supervision of Prof. David Tirrell, at the University of Massachusetts in Amherst. He was appointed in 2000 as a full professor at the Radboud University Nijmegen to set up a group in bio-organic chemistry. His current interests focus on translating natural structural concepts into bio-inspired materials with the aid of state of the art organic and polymeric chemistry techniques and protein engineering. choosing the type of magnetism of the material, ranging from ferromagnetism and paramagnetism to diamagnetism. Second, for each of these magnetic materials, the field strength and orientation can be varied, or kept constant, in space, time or both. We can distinguish homogeneous magnetic fields (field strength and direction constant in space and time), inhomogeneous magnetic fields (strength changes with position but not with time), rotating magnetic fields (direction changes with time), and oscillating magnetic fields (strength changes with time). Third, magnetic fields are a non-invasive way of manipulating matter, because magnetic forces are contactless, volume forces and no chemical alteration is induced. Fourth, when a magnetic field is used for propulsion, no depletion of fuel can occur. Fifth, strong permanent magnets and (superconducting) electromagnets are nowadays readily, commercially, available. Finally, compared to

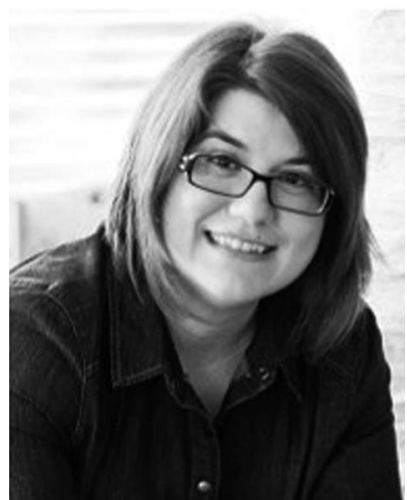

Daniela A. Wilson received her PhD with "summa cum laude" from "Gh. Asachi" Technical University of Iasi, Romania. During her PhD she obtained two fellowships in Japan and $U K$ as an exchange PhD student and a Marie Curie fellow. After two postdoctoral positions at the University of Pennsylvania, Philadelphia, USA and Radboud University Nijmegen, the Netherlands, she joined the Institute of Molecules and Materials in Nijmegen as an assistant professor. In 2012 she was awarded a prestigious ERC starting grant to establish an independent research line investigating nanomotors with autonomous propulsion in biological systems. Her research interests include nanomotors, supramolecular chemistry, drug delivery and synthetic organic chemistry.

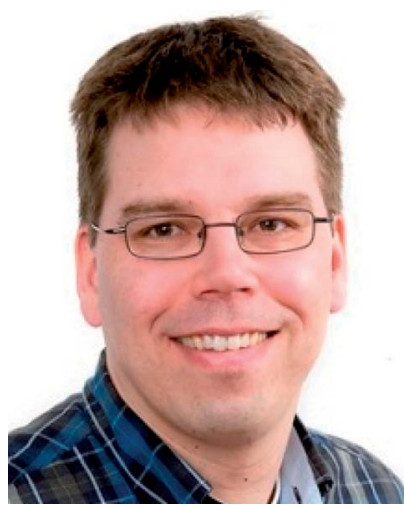

Peter C. M. Christianen received his PhD degree in 1993 at the Radboud University Nijmegen on a thesis about the ultrafast optical properties of semiconductor nanostructures. He started working at the High Field Magnet Laboratory (HFML) of the Radboud University, investigating semiconductor nanostructures using high magnetic fields and optical spectroscopy. Around 2000 he expanded his research area towards the magnetic manipulation of molecular materials, such as liquid crystals, molecular aggregates and polymers, using magnetic alignment and levitation. In September 2013 he was appointed as a full professor at the HFML to investigate the properties of soft condensed matter and nanomaterials in high magnetic fields. 
electric fields magnetic fields do not induce currents by accelerating charged particles in solution ${ }^{\mathbf{1 7}}$ and are not as sensitive to surface charges and $\mathrm{pH} .^{2}$

Although some excellent reviews on magnetic manipulation of nanostructures have been published in the past, ${ }^{17-19}$ most of them have focussed on micro- and nanostructures containing ferromagnetic and paramagnetic particles, since these only require magnetic fields up to several tenths of $\mathrm{mT}$. Here, we also include the magnetic manipulation of diamagnetic materials, which normally requires magnetic fields in the order of $1 \mathrm{~T}$ or more. The ability to magnetically manipulate seemingly nonmagnetic (diamagnetic) materials can lead to new opportunities to control and steer (biocompatible) soft matter. It is our goal to inform the reader about the possibilities of these different types of magnetic materials and magnetic fields for propulsion and steering of micro- and nanostructures.

\section{Magnetism}

In principle magnetic fields can be produced by free electric currents or by magnetic materials, like permanent magnets. In electromagnetism these two different sources of magnetic field $\vec{B}$ are distinguished: ${ }^{20}$

$$
\vec{B}=\mu_{0}(\vec{H}+\vec{M}) .
$$

The field as a result of $\vec{H}$ is generated by a free (externally controllable) current, $\uparrow$ like in the coil of an electromagnet. Magnetic fields produced by magnetic materials are the result of the so-called magnetization $\vec{M}$ (magnetic moment per volume) inside the material. $\mu_{0}$ is the magnetic permeability of vacuum.

Magnetic materials can be divided into several different classes of which the most common are paramagnets, diamagnets and ferromagnets. ${ }^{20}$ In this review we will focus mainly on these three classes. We shall begin with defining paramagnetism and diamagnetism, since they can be described in a similar formalism. After that, ferromagnetism and, shortly, superparamagnetism will be introduced.

\subsection{Basic formalism of paramagnetism and diamagnetism}

We consider para- and diamagnets under experimental conditions (ambient temperature, field strengths) in which a magnetization is induced that is linear with an applied magnetic field: ${ }^{20}$

$$
\vec{M}=\chi \vec{H}
$$

Here $\chi$ is the dimensionless (volume) magnetic susceptibility, where the sign of $\chi$ determines whether a material is para- or diamagnetic: $\chi>0$ for paramagnetic and $\chi<0$ for diamagnetic materials. This means that the direction of $\vec{M}$ is parallel to $\vec{H}$ for paramagnetic materials and antiparallel to $\vec{H}$ for diamagnetic materials. Note that the magnetization is induced by the

$\dagger$ Actually, $\vec{H}$ is determined by the free electric current according to: $\oint \vec{H} \cdot \mathrm{d} \vec{l}=I_{\mathrm{f}}$, the integral of $\vec{H}$ along path $\mathrm{d} \vec{l}$ is equal to the enclosed free current $I_{\mathrm{f}}$. external field and reduces to zero when this field is removed, unlike the ferromagnetic case, which is discussed in Section 2.3. Combining eqn (1) and (2) gives

$$
\vec{B}=\mu \vec{H}
$$

with $\mu \equiv \mu_{0}(1+\chi)$ being the magnetic permeability of the material. For paramagnetic materials $\mu>\mu_{0}$, while for diamagnetic materials $\mu<\mu_{0}$. However, in general para- and diamagnetism is a weak effect and typically $|\chi| \ll 1$, which implies that the effect of $\vec{M}$ on the total field is small: $\vec{B} \approx \mu_{0} H$. Nevertheless, some quite interesting effects can occur when a para- or diamagnetic object is placed in an external field.

The magnetic energy $E$ of an object with volume $V$ is given by $^{21}$

$$
E=-\frac{1}{2 \mu_{0}} \chi V B^{2}
$$

This equation shows that the energy of a paramagnet decreases with magnetic field, while that of a diamagnet increases with magnetic field. Since force and energy are related by $\vec{F}=-\vec{\nabla} E$, the magnetic force can be written as ${ }^{21}$

$$
\vec{F}=\frac{\chi}{\mu_{0}} V(\vec{B} \cdot \vec{\nabla}) \vec{B}
$$

with $\vec{\nabla} \cdot \vec{B}$ being the magnetic field gradient. From this equation it follows that paramagnets are attracted to regions of high magnetic field strength (high field seekers), while diamagnets are expelled from them (low field seekers). This is the main difference between para- and diamagnets.

The most common cause for paramagnetism is the presence of unpaired electrons in atomic or molecular orbitals. ${ }^{22}$ Since electrons have a fundamental property called spin, which can be considered as a magnetic dipole having a permanent magnetic moment $\vec{m}$, these spins will orient parallel to the magnetic field to minimize its energy, given by ${ }^{23}$

$$
E=-\frac{1}{\mu_{0}} \vec{m} \cdot \vec{B}
$$

In materials where all electrons are paired in atomic or molecular orbitals (spin up and spin down), all spins cancel at all times and no net magnetization can be induced. Unpaired electrons however do not have this restriction and can, therefore, contribute to the magnetization of the material. In the absence of an external magnetic field the unpaired spins point in random directions, thereby cancelling each other on a macroscopic level and the total magnetization is zero. When an external magnetic field is applied, the unpaired spins will start to align, leading to a net magnetization. This alignment competes with thermal motion that tends to randomize the spin orientations, which indicates that the paramagnetic response strongly depends on temperature. At low temperatures it is possible to fully align the spins at high magnetic fields and saturate the magnetization. However, at room temperature and for magnetic field strengths typically applied in the research described in this review, the magnetization remains linear with the applied magnetic field, following eqn (2). 
A historic overview of diamagnetism and its discovery by Anton Brugmans is given by Küstler. ${ }^{24}$ Most often, diamagnetism is caused by the tiny distortion of the electron orbits within atoms or molecules by the applied magnetic field. Magnetic dipoles are induced that oppose the external field. ${ }^{25}$ This quantum mechanical effect occurs in all materials but its absolute value is normally smaller than for para- and ferromagnetism. A diamagnetic response is, therefore, observed for materials that do not exhibit para- and ferromagnetism, which is roughly $95 \%$ of all matter. It also means that stronger magnetic fields ( $>1 \mathrm{~T}$ ) are necessary to efficiently manipulate (steer, align or propel) diamagnetic structures in a diamagnetic solution. Although historically this has been relatively hard to achieve, producing such high magnetic fields is no longer a limiting factor. Superconducting magnets up to $20 \mathrm{~T}$ are commercially available and in many cases permanent neodymium-iron-boron magnets are also sufficient.,2,24-28 Since the vast majority of (soft) materials are diamagnetic, this creates new opportunities to manipulate those materials with magnetic fields, including many biomolecules, such as DNA, RNA, peptides and the majority of cells and tissues, ${ }^{29}$ indicating that diamagnetic manipulation can be employed in biological and medical research.

\subsection{Manipulation of paramagnets and diamagnets}

Manipulation of para- and diamagnets can be done in homogeneous or inhomogeneous magnetic fields. Homogeneous magnetic fields are frequently used to align these magnetic materials. Two mechanisms are available that allow magnetic orientation of paramagnetic and diamagnetic particles. ${ }^{21}$ The first one is due to an intrinsic anisotropy of the magnetic susceptibility of the material, which occurs when $\chi$ differs between at least two axes. The most simple case is a uniaxial material, with a susceptibility difference of $\Delta \chi$ between the two main axes, leading to the following angle dependence of the magnetic energy: ${ }^{25}$

$$
E(\theta)=-\frac{1}{2 \mu_{0}} \Delta \chi V B^{2} \cos ^{2} \theta
$$

Here, $\theta$ is the angle between the axis of the lowest susceptibility and the applied magnetic field. These materials will orient in such a way that their axis corresponding to the highest susceptibility is parallel to the magnetic field.

The second method to align para- and diamagnetic materials is via so-called shape anisotropy. ${ }^{21}$ The induced magnetic moment (magnetization integrated over volume) not only depends on the magnetic susceptibility, but also on the shape of the object, due to the so-called demagnetization effect (see Fig. 1). As a consequence, it is energetically favourable to align the object with its longest axis parallel to the magnetic field. ${ }^{21}$

Inhomogeneous magnetic fields can be used to propel microand nanostructures in solution as indicated by eqn (5). Since in soft matter most structures are in solution, one has to account for the magnetic susceptibility of the solvent as well. In this case, it is the difference in magnetic susceptibility between the solvent and the object that determines the magnetic force: ${ }^{30}$

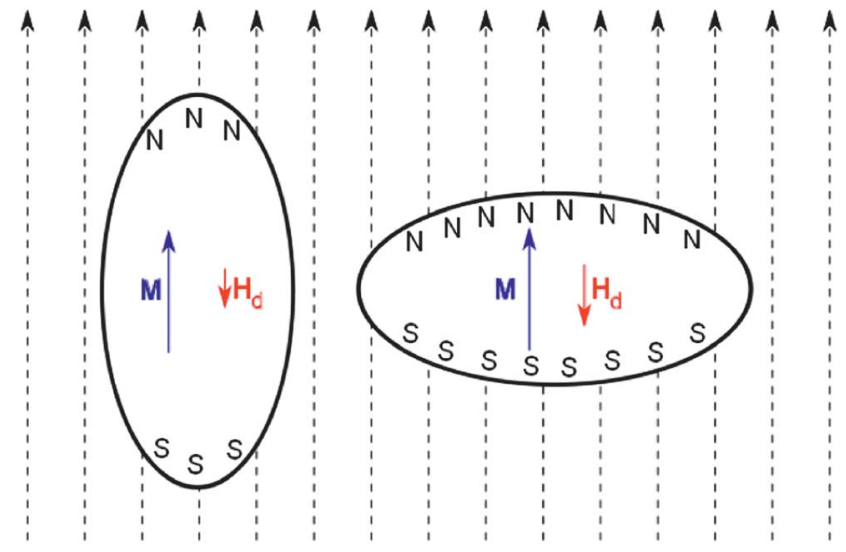

Fig. 1 Two identical pieces of paramagnetic material that are magnetized along two different axes by an external magnetic field (dashed arrows). Left: the long axis of the paramagnet is aligned along the external field. At the ends magnetic dipole charges (indicated by $\mathrm{N}$ and S) are induced leading to a demagnetizing magnetic field $H_{d}$ antiparallel to the induced magnetization. Right: when the paramagnet is aligned with its long axis perpendicular to the external field, the induced demagnetizing field is larger, due to the enhanced magnet surface charges. Alignment along the long axis results in the smallest demagnetizing field and is therefore energetically favourable.

$$
\vec{F}=\frac{1}{\mu_{0}}\left(\chi_{\text {obj }}-\chi_{\text {sol }}\right) V(\vec{B} \cdot \vec{\nabla}) \vec{B}
$$

with $\chi_{\mathrm{obj}}-\chi_{\text {sol }}$ being the difference in magnetic susceptibility between the object and the solvent. From this equation it follows that the force on a diamagnetic object (negative $\chi$ ) can be enhanced by placing it in a paramagnetic solution (positive $\chi)^{26,27,31-34}$

Diamagnetic materials are repelled by a magnetic field, which allows the stable magnetic levitation in a field gradient, at a position in the magnet where the magnetic force balances the gravitational force. ${ }^{35}$ Braunbek was the first to demonstrate diamagnetic levitation by placing graphite and bismuth in an inhomogeneous magnetic field created by an electromagnet. ${ }^{24}$ Fifty years later, Beaugnon and Tournier demonstrated the levitation of other weaker diamagnetic materials such as water, wood and plastics using a hybrid magnet, composed of a Bittertype electromagnet and a superconducting magnet. ${ }^{36}$ Strong Bitter and hybrid magnets can provide these gradients over volumes on the order of $\mathrm{cm}^{3}$. Indeed, it was demonstrated that (bio)matter containing large amounts of water (such as a living frog) could be levitated. ${ }^{37}$ Water microdroplets have also been levitated using permanent neodymium-iron-boron magnets, which proves that these strong gradients can also be created by permanent magnets albeit over much smaller volumes. ${ }^{28}$

Paramagnetic solutions or gasses have been used to lower the necessary $(\vec{B} \cdot \vec{\nabla}) \vec{B}$ required for levitation. ${ }^{31,33,34,38}$ In such a case the forces acting on the diamagnetic particles are determined by the buoyancy and the difference in magnetic susceptibility per unit of volume between the diamagnetic object and the paramagnetic solution: ${ }^{21}$

$$
\frac{\vec{F}}{V}=-\left(\varrho_{\text {dia }}-\varrho_{\text {sol }}\right) \vec{g}+\frac{1}{\mu_{0}}\left(\chi_{\text {dia }}-\chi_{\text {sol }}\right)(\vec{B} \cdot \vec{\nabla}) \vec{B},
$$


with $\vec{F} / V$ being the force on the diamagnetic object per unit of volume, $\varrho_{\text {dia }}-\varrho_{\text {obj }}$ the difference in density between the diamagnetic object and the solvent, $\vec{g}$ the specific gravity and $\chi_{\text {dia }}-\chi_{\text {sol }}$ the difference in magnetic susceptibility between the diamagnetic object and the solvent. Levitation occurs when $\vec{F} / V$ equals zero and is known as Magneto-Archimedes levitation when a paramagnetic solution is employed to facilitate the levitation.

\subsection{Ferromagnetism}

Ferromagnetism is the most familiar type of magnetism, since it is responsible for permanent magnetism. It occurs for materials with unpaired electrons, which have a strong mutual interaction and a positive exchange energy. ${ }^{22}$ As a consequence it is energetically favourable for adjacent spins to align parallel and form domains. Within each domain the spins point in the same direction, but this direction varies amongst different domains. In an unmagnetized sample the domains are randomly oriented, so the net magnetization is zero. By applying a magnetic field the domains tend to align along the field direction: the domains that are originally aligned along the field grow at the expense of the others. When the external field is removed the net magnetization does not return to zero, which is exploited in the fabrication of permanent magnets.

The magnetization of a ferromagnet is thus highly nonlinear and depends on the history of the sample and the applied fields (magnetic hysteresis), as is shown in Fig. 2. The figure shows the applied magnetic field $\mu_{0} H$ on the $x$-axis and the total magnetic field $B$ or magnetization $M$ on the $y$-axis. For ferromagnets, $B$ is predominantly given by $M$, which is much larger than $H$.

When starting with unmagnetized material $\left(B=\mu_{0} H=0\right)$, applying a magnetic field leads to the dashed curve in Fig. 2. The magnetization saturates (beyond $\mu_{0} H_{\mathrm{S}}$ ) when all domains point in the same direction. When the applied field is reduced to zero, the ferromagnet will retain a net magnetization. One needs to apply a magnetic field in the opposite direction to bring the magnetization back to zero. The field necessary to flip

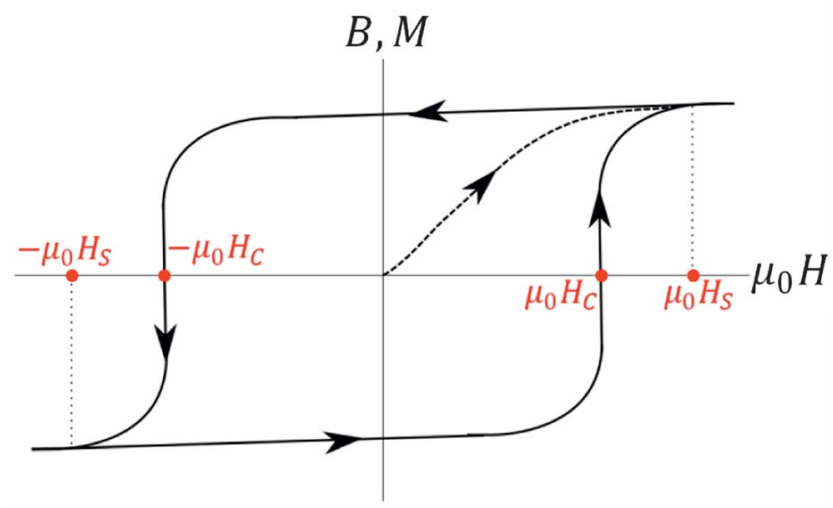

Fig. 2 Representation of a typical magnetization hysteresis loop for a ferromagnet. $\mu_{0} H_{C}$ is the coercive magnetic field, which is the applied field at which the total magnetic field $B$ or magnetization $M$ changes sign. $\mu_{0} H_{S}$ is the applied magnetic field at which the magnetization saturates. the direction of magnetization is called the coercive field $H_{\mathrm{c}}$. A magnetized ferromagnet maintains its magnetization as long as the applied magnetic field is smaller than this coercive field. In this case, a ferromagnet will orient with its magnetic moment parallel to the applied magnetic field (eqn (6)). Note the difference with the magnetization of para- and diamagnets that are linear with the applied magnetic field at ambient temperatures (eqn (3)).

According to eqn (6), the energy of a permanent magnetic dipole decreases with increasing magnetic field. Since force and energy are related by $\vec{F}=-\vec{\nabla} E$, the magnetic force in an inhomogeneous field can be written as $^{3}$

$$
\vec{F}=\frac{1}{\mu_{0}}(\vec{m} \cdot \vec{\nabla}) \vec{B},
$$

Eqn (10) shows that a ferromagnetic particle in an inhomogeneous field will experience a translational force towards regions of higher magnetic field. The strength of this force scales linearly with the size of both the magnetic dipole moment and the field gradient. Again it must be noted that eqn (10), like eqn (6), is valid as long as the applied magnetic field is below the coercive field of the material.

\subsection{Superparamagnetism}

Superparamagnetism occurs for very small single domain ferromagnets (or ferrimagnets:). In this case, the thermal energy is able to constantly turn over the direction of the magnetization. As a result, the average magnetization is zero and the material behaves as a paramagnet. However, since the particle still consists of a single domain, its susceptibility for magnetic fields is much larger than that of a normal paramagnet, hence its classification as a superparamagnet. Since superparamagnets behave similarly to normal paramagnets, we discuss superparamagnetic particles in the paramagnetism section. A more elaborate discussion of superparamagnetic particles can be found in ref. 39 .

In the remainder of this review, we will focus on the three introduced classes of magnetic materials. For each, we will discuss how homogeneous, inhomogeneous, rotating and oscillating magnetic fields can be used to manipulate the motion of micro- and nanostructures. Manipulation of motion consists of propulsion, steering, or a combination of the two. An overview of these combinations is shown in Table 1.

\section{Structures containing ferromagnetic materials}

\subsection{Homogeneous magnetic fields and ferromagnets}

Several groups have used ferromagnetic particles to enable steering of their micro- and nanostructures. Kline et al. have

$\ddagger$ Ferrimagnets consist of domains like ferromagnets, but within each domain the spins have an alternating up and down orientation. Since the up and down spins are created by different species or ions within the crystal lattice, the magnetic moments of the two are not of equal size and hence do not cancel completely. Each domain therefore maintains a total magnetic moment unequal to zero. 
Table 1 Overview of magnetic materials, magnetic fields and applications

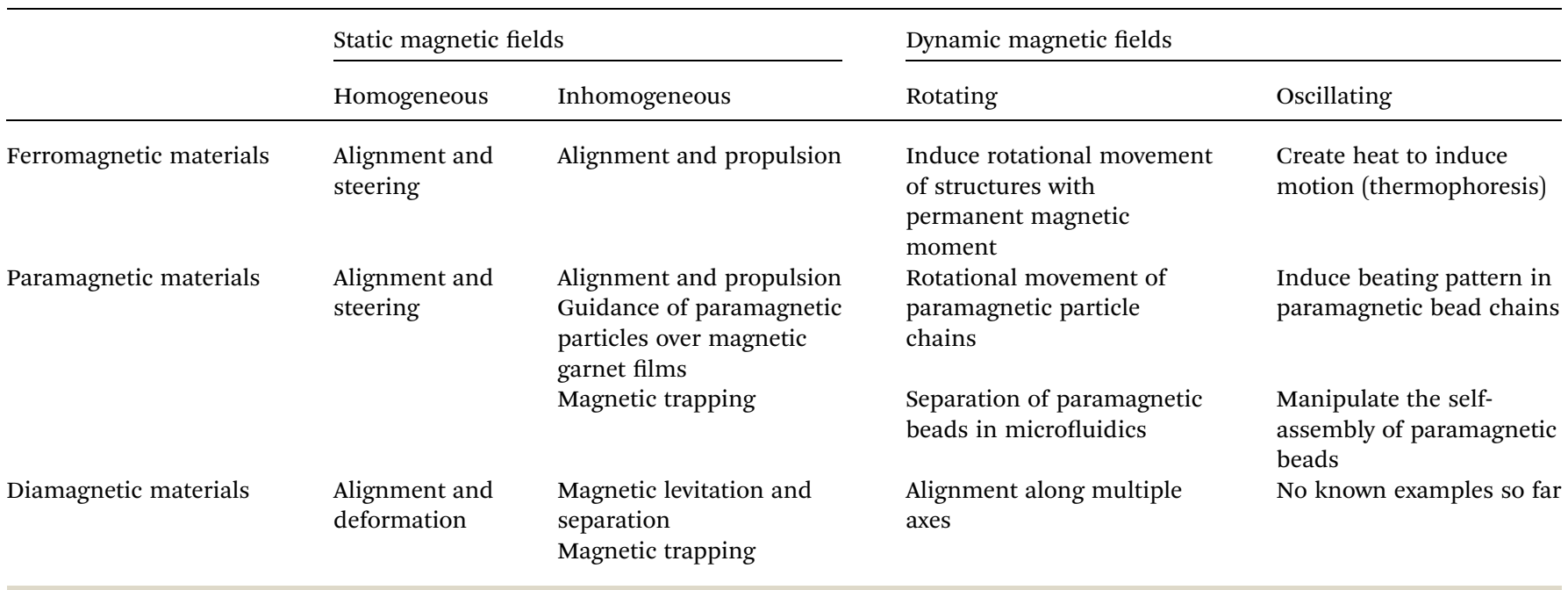

fabricated 1.5 micron long rods consisting of two alternating nickel and gold domains, which were capped by a platinum domain at one end. ${ }^{\mathbf{4 0}}$ Propulsion was achieved chemically by catalytic decomposition of hydrogen peroxide on the platinum surface. This led to the formation of oxygen bubbles, which gave the rod a recoil upon dissociation from the platinum surface and hence led to the desired propulsion. ${ }^{41}$ The nickel sections were magnetized perpendicularly to the rod, ensuring a perpendicular alignment of the rod relative to the magnetic field direction (see Fig. 3). Steering was achieved by changing the direction of an applied magnetic field of only $55 \mathrm{mT}$.

This combination of magnetic steering and chemical propulsion has been explored further by other groups. Baraban et al. made Janus motors by partly capping $5 \mu \mathrm{m}$ silica particles with a cobalt layer used for magnetization, which was topped with a platinum layer for catalysis of hydrogen peroxide decomposition. ${ }^{\mathbf{4 2}}$ The Janus motors were steered by magnetic fields below $1 \mathrm{mT}$. Other examples include microtubes made of rolled-up titanium-iron-platinum films, which decomposed hydrogen peroxide inside the tube. ${ }^{6}$ The propulsion resulted from the expulsion of oxygen bubbles at the largest opening of
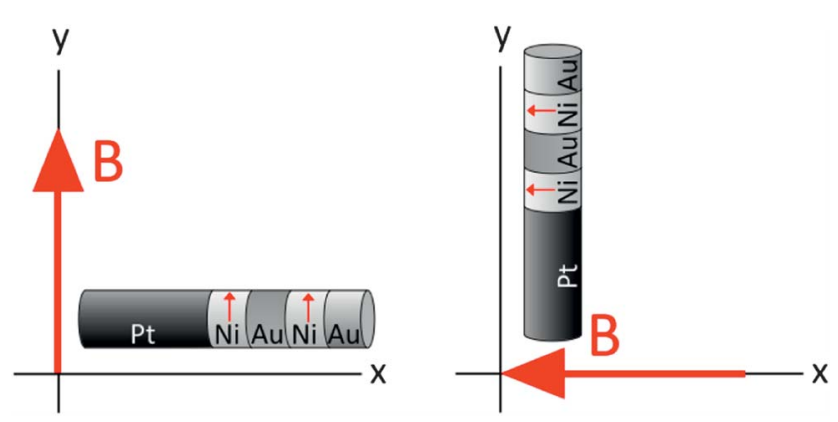

Fig. 3 Magnetic steering of rods consisting of Pt, Ni and Au segments. Rotation of the magnetic field leads to the rotation of the nanorod, such that the long axis of the rod is perpendicular to the field direction. The magnetic moments in the nickel segments are indicated by the small arrows. Adapted from ref. 41. the microtube. The presence of the ferromagnetic iron provided the advantage of facile manipulation and steering of the tubes by magnetic fields of $5 \mathrm{mT}$. Furthermore these tubes were able to transport and organise nanoplates.

\subsection{Inhomogeneous magnetic fields and ferromagnets}

Gradient magnetic fields were used in many occasions to propel ferromagnetic micro- and nanostructures. For instance, it was shown that polymer beads coated with ferromagnetic nickel could be translated in a horizontal direction by a magnetic field gradient. ${ }^{43}$ Movement in the vertical direction was determined by buoyancy created by oxygen bubbles upon hydrogen peroxide decomposition on the nickel surface.

Similar experiments were also performed with cobalt ferrite microparticles, which were doped with palladium. ${ }^{44}$ In a solution of hydrogen peroxide, propulsion of the particles was determined by oxygen formation on the particle surface. The authors demonstrated that small magnetic fields could be used to align and hence steer the ferromagnetic particles. However, higher magnetic fields also led to higher magnetic field gradients, which caused the microparticles to aggregate at the magnetic poles, where the magnetic field was strongest. It was also mentioned that relatively large particles, up to $150 \mu \mathrm{m}$ in diameter, were used in order to track their movement with optical microscopy. Many examples in the literature use micrometer-sized structures to allow for observation using optical microscopy and to avoid motion disruptions caused by Brownian motion, which occurs when the structures become so small that their magnetic energy becomes smaller than the thermal energy. Besides optical microscopy, only a few other in situ techniques are available to track magnetic particles. In situ dynamic light scattering ${ }^{45}$ or in situ confocal microscopy ${ }^{\mathbf{4 6}}$ are suitable alternatives, which also have the potential of tracking nanometer-sized objects. The problem of decreasing magnetic energy upon miniaturization of magnetic objects is resolved by the employment of higher magnetic fields (i.e. superconducting or Bitter magnets). ${ }^{47}$ 
The use of magnetic field gradients has also shown promising for applications in drug delivery. Mathieu et al. have investigated the possibility whether ferromagnetic particles can be steered through blood vessels by the magnetic field gradients in a MRI machine. ${ }^{48-50}$ For this purpose, carbon steel spheres (with 2.38 and $3.18 \mathrm{~mm}$ diameters) were forced to move against a water flow by a magnetic field gradient of $18 \mathrm{mT} \mathrm{m}^{-1}$ created within a MRI magnet. The flow properties were chosen to be close to those in real arteries. Since the employed particles are rather large (mm range) they could only find applications in the larger blood vessels like the aorta. The authors underlined that for propulsion of smaller particles through real mice capillary gradients of several $\mathrm{T} \mathrm{m}^{-1}$ would be needed. Since MRI systems cannot provide such large gradients additional gradient coils are needed. A coil providing a gradient of $0.443 \mathrm{~T} \mathrm{~m}^{-1}$ proved to be sufficient to move and steer $10.82 \mu \mathrm{m}$ iron oxide microparticles in a simple artificial Y-shaped channel though. ${ }^{50}$

More examples on biomedical applications of inhomogeneous magnetic fields are summarized in the reviews by Pankhurst et al.51,52 They offer excellent overviews on topics like magnetic separation, using magnetic particles, and magnetically guided drug delivery.

\subsection{Rotating magnetic fields and ferromagnets}

When a magnetic dipole is exposed to a magnetic field, it will align along the magnetic field according to eqn (6). This principle can be utilized to induce rotational motion in a structure containing a permanent magnetic dipole by rotating the applied magnetic field. This can be achieved by rotating a bar magnet or by setting up two perpendicular coils through which an oscillating current is fed. The strength of the rotating field should be smaller than the coercive field of the ferromagnet. Fields of a few $\mathrm{mT}$ are usually sufficient. ${ }^{53-59}$

Magnetically induced rotations are often converted into translational motion, ${ }^{53-58}$ but also the investigation of the rotation itself has been reported. ${ }^{59}$ Translational movements of micro- and nanostructures induced by rotating magnetic fields are often inspired by bacterial flagella. ${ }^{53-56}$ Honda et al. were the first to investigate if a helical structure coupled to a permanent magnet could be propelled by a rotating magnetic field in the microworld. ${ }^{53}$ Their construct consisted of a 1 $\mathrm{mm}^{3}$ magnet coupled to a thin helical copper wire of several centimetres long. Rotation of the applied magnetic field resulted in the rotation of the small magnet and the helix attached to it, which propelled the whole structure forward. The direction of the motion inverted by reversing the rotation direction of the magnetic field. The structure was placed inside high viscosity silicone oil to emulate low Reynolds numbers, similar to the condition for microstructures in water, and operated at frequencies between 2 and $5 \mathrm{~Hz}$ for the most viscous oil used.

A similar structure, but on the micron scale, was made by Zhang et $a .^{54}$ An artificial bacterial flagella (ABF), $47 \mu \mathrm{m}$ long, was fabricated by connecting a magnetic head consisting of a $\mathrm{Cr} / \mathrm{Ni} / \mathrm{Au}$ trilayer square plate to a helical tail made of an InGaAs/GaAs bilayer or an InGaAs/GaAs/Cr trilayer. Three pairs of orthogonal coils were used to rotate a magnetic field in all possible directions. Rotation of the applied magnetic field at 1-1.67 Hz resulted in rotation of the magnetic square plate and hence the helical tail as shown in Fig. 4. Rotation of the helical tail propelled the ABF forward or backward, depending on the chirality of the helix. Furthermore, the direction of propulsion could be inverted by rotating the magnetic field in the opposite direction. Steering of the ABF was achieved by alignment of one of the magnetic plate diagonals with the magnetic field. Other examples utilizing rotating magnetic fields to induce linear movement include the linkage of a polystyrene microbead to a magnetic nanoparticle with an actual flagellar filament, ${ }^{55}$ planar ferromagnetic polymer structures, which deformed into helical structures when a rotating magnetic field was applied, ${ }^{56}$ and DNA-linked paramagnetic particles on glass surfaces. ${ }^{57}$

A $7 \mu \mathrm{m}$ long rodlike hybrid motor was reported by Gao $e t$ al., which consisted of sequential nickel, silver, gold and platinum domains. ${ }^{58}$ The nanowire could be propelled either by decomposition of hydrogen peroxide at the gold/platinum end, or by applying a rotating magnetic field which led to the rotation of the ferromagnetic nickel (see Fig. 5). The silver was made flexible by partly dissolving it in a hydrogen peroxide solution, which allowed the breaking of the symmetry of the rod, enabling the transfer of rotation into translational motion. Since the two forms of propulsion acted in opposite directions, a change in the propulsion method also led to a change in direction.

\subsection{Oscillating magnetic fields and ferromagnets}

While rotating magnetic fields were widely used to induce direct rotational motion, which is often transformed into translational motion, oscillating magnetic fields were used to induce heat in ferromagnetic materials. This technique is investigated in the medical world as a possible treatment for cancer, where it is known as magnetic hyperthermia. ${ }^{60-63}$ The magnitude of magnetic hyperthermia is related to the energy needed to change the magnetization, which is equal to the area under the magnetization hysteresis curve. By applying an oscillating magnetic field, the magnetization will change continuously in

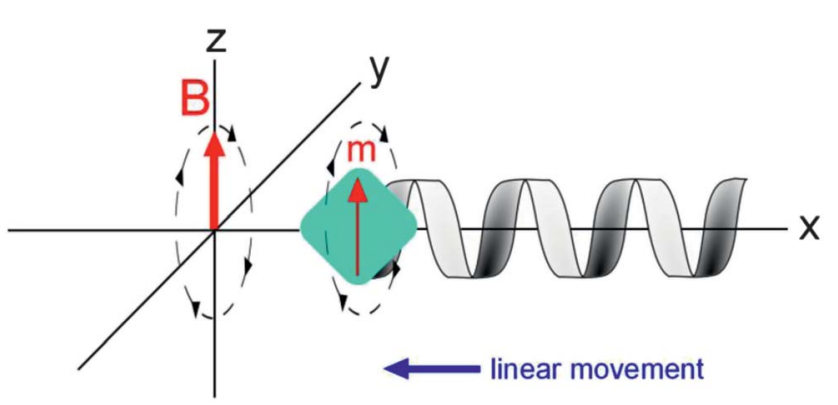

Fig. 4 Helix attached to a ferromagnet in a rotating magnetic field. The magnetic moment of the ferromagnet constantly aligns along the magnetic field, leading to rotation of the ferromagnet and the helix. Rotation of the helix will induce linear motion, in this case along the $-x$ direction. Adapted from ref. 54 


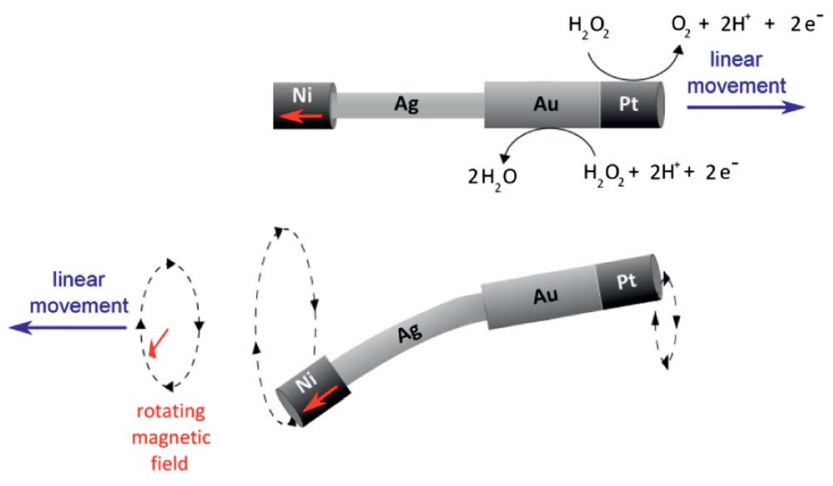

Fig. 5 Hybrid nanowire showing the two methods of propulsion. Top: catalytic propulsion by hydrogen peroxide decomposition. Bottom: magnetic propulsion by a rotating magnetic field. The magnetic moments in the nickel segments are indicated by the small arrows. Adapted from ref. 58.

direction and magnitude, following the magnetization hysteresis curve like the one shown in Fig. 2. The energy of the oscillating magnetic field, which is essentially electromagnetic radiation, is absorbed in this process by the ferromagnet and is dissipated in the form of heat. The amount of heat generated can be controlled by both the frequency and the amplitude of the oscillating magnetic field. ${ }^{62}$

Propulsion of microstructures using the concept of magnetic hyperthermia has recently been demonstrated by Baraban et al. ${ }^{63}$ Micron sized silica particles were coated on one side with a magnetic permalloy. Frequencies of $400-6000 \mathrm{~Hz}$ with an amplitude of $7.5 \mathrm{mT}$ were applied by a solenoid coil. Local heating of the magnetic cap provided a temperature gradient in the solvent, which propelled the Janus particle in the direction opposite to the magnetic cap by thermophoresis.

\section{Structures containing paramagnetic materials}

\subsection{Homogeneous magnetic fields and paramagnets}

Butykai et al. showed that paramagnetic anisotropic rod-like triclinic heme crystals, called hemozoin, can be magnetically aligned, using magnetic fields up to $5 \mathrm{~T}^{.64}$ The heme crystals consist of hematin dimers linked via an iron-oxygen bond of adjacent hematin molecules, which are assembled via hydrogen bonding. Hemozoin crystals are aligned with their long axis perpendicular to the magnetic field, since that axis has the highest paramagnetic susceptibility.

Alignment of particles based on shape anisotropy has been demonstrated by Tierno et al.$^{65}$ Ellipsoid polystyrene particles, doped with superparamagnetic iron oxide, were oriented with their long axis parallel to the magnetic field. By coating part of the particle surfaces with platinum, the ellipsoids were propelled by the catalytic decomposition of hydrogen peroxide into water and oxygen. Steering of the ellipsoid particles was successfully achieved by changing the orientation of the magnetic field as shown in Fig. 6. Steering of similar particles of spherical shapes could not be achieved, indicating that the shape anisotropy was indeed responsible for the alignment.

Doublet microparticles, consisting of silver and superparamagnetic Dynabead microspheres, were demonstrated by Chaturvedi $e t a l$. to form long chains aligned by magnetic fields generated by neodymium-iron-boron bar magnets ${ }^{66}$ Furthermore, the chains could be decoupled from each other by activating the propulsion of the doublet particles. The propulsion of the doublet particles was not based on oxygen formation as in previously reported systems but the result of diffusiophoresis, which, besides the presence of hydrogen peroxide, also required irradiation with UV light to form silver and $\mathrm{OOH}^{-}$ions.

Since homogeneous magnetic fields do not propel ferroand paramagnetic particles, the use of such fields is normally limited to orienting and steering. However, recent experiments have shown that a homogeneous magnetic field can alter not only the direction but also the speed of Janus particles partially covered with a cobalt/platinum layer topped by palladium. ${ }^{67}$ In a hydrogen peroxide solution, the palladium catalyzes the decomposition of hydrogen peroxide into oxygen and water. During this process $\mathrm{OH}^{-}$and $\mathrm{H}^{+}$ions are formed as intermediates, which recombine into water. The authors claimed that these ions, which are only produced on the palladium side of the Janus particle, created an electric field responsible for polarization of the Janus particle and forced it to move towards the palladium side. This self-electrophoresis counteracts the propulsion created by the oxygen dissociation. The authors suggest that the magnetic field is responsible for an increase in recombination time of the $\mathrm{OH}^{-}$and $\mathrm{H}^{+}$ions by giving the ions a helical component. The helical component is induced by the Lorentz force, acting on moving charged particles in a magnetic field and directed perpendicular to the velocity of the particle and the magnetic field. This then leads to a decrease in the scattering cross-section and hence an increase in the ion lifetimes.

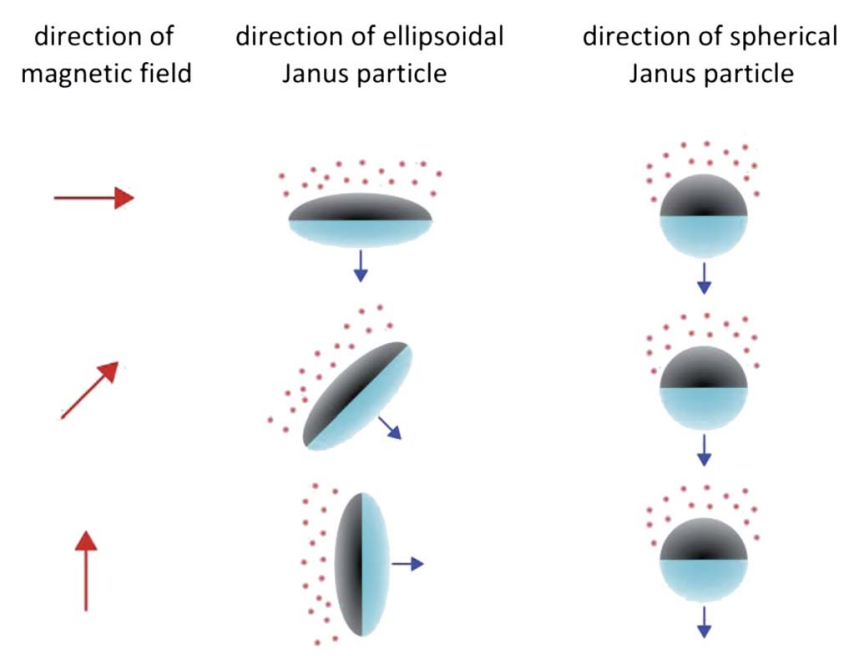

Fig. 6 Alignment of paramagnetic Janus particle coated partly with platinum (black) for decomposition of hydrogen peroxide. Formation of oxygen (small spheres) propelled the Janus particles in the direction indicated by the small arrows. Magnetic steering occurred only when shape anisotropy was present. Adapted from ref. 65. 


\subsection{Inhomogeneous magnetic fields and paramagnets}

The effect of gradient magnetic field forces on different kinds of paramagnetic transition metal ions has been studied by Tanimoto and co-workers. ${ }^{68-71}$ In a gradient of $0.41 \mathrm{~T}^{2} \mathrm{~m}^{-1},((\vec{B} \cdot \vec{\nabla}) \vec{B})$, paramagnetic ions like $\mathrm{Fe}^{3+}, \mathrm{Co}^{2+}, \mathrm{Ni}^{2+}$ and $\mathrm{Cu}^{2+}$ were deposited in droplets on a silica gel plate. The droplets followed the direction of the gradient towards higher magnetic fields. The authors showed that the extent of displacement depended on the value of the paramagnetic susceptibility, the adsorption to the silica gel and the ion concentration of the droplets. ${ }^{69-71}$ Also, the amount of displacement was much larger than that could be explained by the drift velocity of a single ion induced by the magnetic field gradient. These latter results indicate that the ions do not move separately in a magnetic field gradient but rather in an orchestrated cluster consisting of both ions and water molecules. It was suggested that the magnetic force on individual ions was conveyed to ions and the surrounding water molecules by collisions resulting in collective behaviour of paramagnetic ions and water molecules. For $\mathrm{Cu}^{2+}$ the diameter of such a cluster was calculated to be $4.6 \mu \mathrm{m}$. The movement of paramagnetic particles does furthermore depend on the concentration gradient of these particles. Effectively, in an inhomogeneous solution of paramagnetic particles, the susceptibility of the solution as a whole will consequently be inhomogeneous as well. This will lead to magnetoconvection, in which regions of high susceptibility move towards higher fields, replacing regions of lower susceptibility.

Magnetic field gradients of a permanent magnet have also been used to accumulate superparamagnetic iron oxide at one side of ellipsoidal polystyrene particles, leading to the formation of ellipsoidal Janus particles. ${ }^{72}$ The authors further showed that the interaction between such Janus particles differed from the interaction between ellipsoids with homogeneous distributions of iron oxide.

Magnetic field gradients can also be created by magnetic garnet films. ${ }^{73,74}$ These ferromagnetic films contain magnetic domains which have their magnetic moments directed perpendicularly to the plane, pointing either up or down. At the boundary of two domains with opposite magnetic moments, a strong magnetic field gradient exists. Dhar et al. have shown that superparamagnetic particles can easily be guided along such boundaries as shown in Fig. $7 .{ }^{73}$ Since the

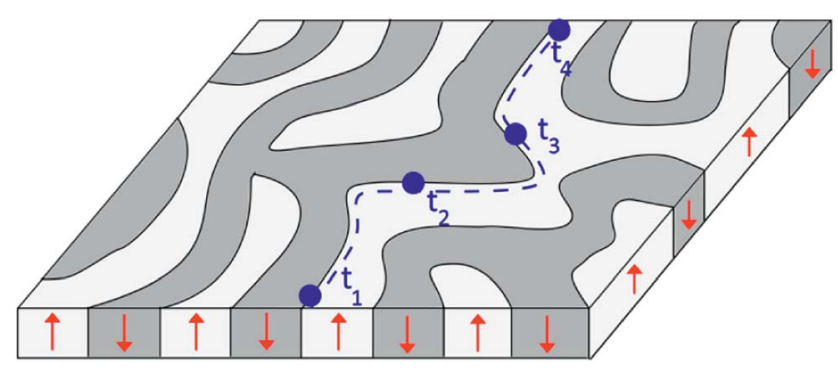

Fig. 7 Representation of a magnetic garnet film. The arrows at the sides show the direction of the magnetic moments. A paramagnetic particle (spheres) can be directed along boundaries of regions with opposite magnetic moments. Adapted from ref. 73. domain sizes and their magnetic orientation (up/down) can be manipulated beforehand, it is possible to create predefined magnetic paths along which paramagnetic particles can be moved..$^{74}$

Superparamagnetic particles in combination with inhomogeneous magnetic fields are also frequently employed in microfluidic applications, often for separation, mixing, and biochemical procedures. For an extensive review on this specific topic we direct the reader to ref. 2-4.

\subsection{Rotating magnetic fields and paramagnets}

It was demonstrated that superparamagnetic microparticles (polystyrene spheres doped with iron oxide) form chains when a magnetic field is applied. ${ }^{75}$ This is caused by the dipole-dipole interactions between the induced dipoles of each microparticle. Since these chains are of anisotropic shape, they can be rotated with a rotating magnetic field. Vuppu et al. achieved this for rotating fields of 4.5-7 $\mathrm{mT}^{76,77}$ At very low frequencies (5 rpm), growth of the chains was observed. This was explained by the fact that rotation increases encounters between different chains. Higher frequencies led to breakage of chains, induced by viscous forces.

To prevent the breakage of the chains, Biswal et al. chemically linked the beads with glutaraldehyde to create rigid chains and with poly(ethylene glycol) (PEG) to create flexible chains. ${ }^{78,79}$ The stiffness of the PEG-linked chains was adjusted by varying the length of the PEG linker. Flexible chains were shown to deform under rotating fields into a bent or even folded conformation (see Fig. 8).

Rotating magnetic fields were not only used to induce rotation of paramagnetic chains. Karle et al. used a rotating permanent magnet to pull superparamagnetic beads labelled with DNA from one channel to the other in a microfluidic device. ${ }^{80}$ The rotating magnet created an alternating strong and weak magnetic field. While the strong (inhomogeneous) field was responsible for pulling the paramagnetic particles from one

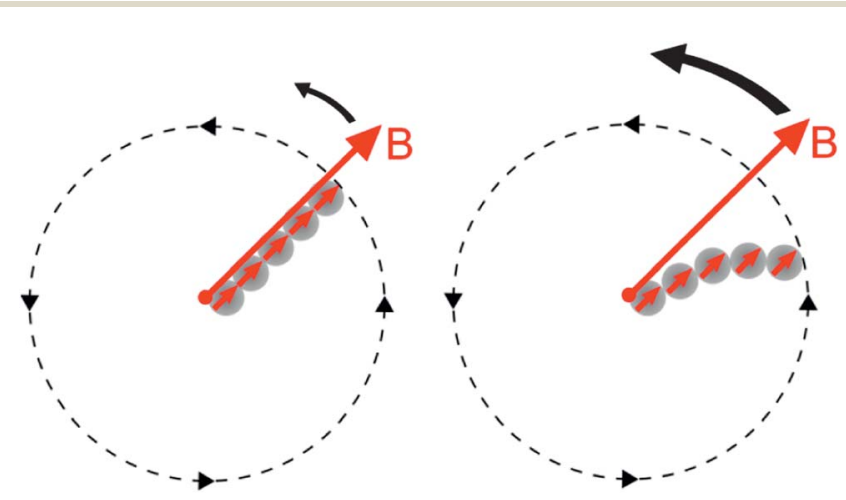

Fig. 8 Rotation of a chain of paramagnetic particles (PEG-linked) induced by a rotating magnetic field. Left: at low rotation frequencies the chain of particles will follow the magnetic field without a lag phase. Right: at high rotation frequencies there will be a lag phase between the chain and the magnetic field. At sufficiently high frequencies the chain will break. The small arrows in the paramagnetic particles represent the induced magnetization. Adapted from ref. 78. 
channel to the other, the weak field was necessary to prevent the particles from sticking to the channel's surface, which would have prevented further movement along with the flow.

The same group also demonstrated how a rotating magnetic field can be used to allow a superparamagnetic bead chain to walk over a microchannel's inner surface. ${ }^{81}$ Walking of bead chains was based on alignment of the bead chain by the magnet (which changed its orientation by rotation) and friction on the channel's surface caused by attraction of the bead chain by the inhomogeneous magnetic field. The authors demonstrated that the bead chain could even walk against flow of up to $7.9 \mathrm{~mm} \mathrm{~s}^{-1}$.

\subsection{Oscillating magnetic fields and paramagnets}

Dreyfus et al. have attached paramagnetic bead chains, interconnected with DNA linkers, to red blood cells. ${ }^{82}$ These structures were exposed to a combination of a static homogeneous magnetic field and a perpendicular oscillating magnetic field, both on the order of $10 \mathrm{mT}$. The total magnetic field therefore changes orientation as a function of time and this led to an induced beating pattern of the paramagnetic bead chain similar to that of a eukaryotic flagella. This induced beating pattern propelled the red blood cells in the direction of the paramagnetic chain. The authors demonstrated that the velocity of the red blood cells depends on parameters like the length and rigidity of the bead chain, the oscillation frequency and strength of the static magnetic field component.

A combination of an oscillating magnetic field with a static homogenous magnetic field was also used to influence the selfassembly of superparamagnetic beads. ${ }^{83}$ The authors demonstrated that at frequencies below $3 \mathrm{~Hz}$, bead chains with $\mathrm{L}$ and $\mathrm{T}$ junctions were created. The distance between junctions could be increased by increasing the frequency. Above $3 \mathrm{~Hz}$, the changing of the induced magnetic dipole in the beads was too fast compared to the translational movement. This led to the formation of dislike structures, originating at the places of highest bead concentration, which was near the junctions.

\section{Diamagnetic structures}

\subsection{Homogeneous magnetic fields and diamagnets}

Alignment of diamagnetic materials in magnetic fields has already been demonstrated for several molecules or molecular structures. ${ }^{84-88}$ Magnetic alignment can greatly enhance the orientational order within a material, thereby improving several of its properties. Depending on the diamagnetic molecules or clusters used, magnetic alignment has for instance already been used to improve optical, ${ }^{85}$ transport ${ }^{86}$ and electrical properties. ${ }^{87,88}$

A well known example of steering diamagnetic materials is the alignment of Paramecium Caudatum, a unicellar organism which is diamagnetically anisotropic. ${ }^{\mathbf{8 9}}$ It was demonstrated that in fields larger than $3 \mathrm{~T}$, swimming paramecium could be aligned, and hence their motion could be steered. The authors emphasised the difference with magnetotactic bacteria, which contains permanent magnetic structures called magnetosomes that allow sensing of the Earth's magnetic field of $5 \times 10^{-5} \mathrm{~T}$.

Alignment of diamagnetic molecules has also been used to deform structures assembled from diamagnetic molecules. ${ }^{25} \mathrm{~A}$ well-known example is the deformation of liposomes. Phospholipids are diamagnetically anisotropic and tend to align perpendicular to a magnetic field. Since liposomes are made from phospholipids, a magnetic field forces the molecules to reorient, deforming the spherical liposome. This magnetic deformation force is balanced by the increasing bending force in the phospholipid membrane. Helfrich was the first to develop a model for the deformation of lipid bilayer vesicles. ${ }^{\mathbf{9 0 , 9 1}}$ Helfrich's original idea was to use the magnetically induced deformation to determine the flexibility of these vesicles. The model assumed deformation of spheres into ellipsoids.

Helfrich's models have been used by other groups in which actual experiments were carried out to deform diamagnetic vesicles in homogeneous magnetic fields. ${ }^{92-95}$ For example, it has been shown that liposomes made from dipalmitoylphosphatidyl choline (DPPC) can be deformed at fields of $3.8 \mathrm{~T}$ as a function of temperature. ${ }^{92} \mathrm{~A}$ transformation in shape from spherical to an elongated sphere (spheroid) was observed at temperatures higher than the glass transition temperature by measuring the magnetically induced birefringence. These kinds of liposomes were also reported to fuse under the influence of magnetic fields of up to $28 \mathrm{~T} .^{93}$

Deformation is not limited to DPPC liposomes. For instance, a NMR study on dimyristoylphosphatidylcholine (DMPC) vesicles also showed deformation at $11.7 \mathrm{~T} .{ }^{94}$ With small angle neutron scattering, deformation was even already observed at magnetic fields up to $4 \mathrm{~T} .^{95}$ In both cases, the extent of deformation was increased significantly above the glass transition temperature.

Tan et al. have shown that the orientation of phospholipid bicelles in a magnetic field can be switched from a parallel to a perpendicular orientation by adding biphenyl moieties to the phospholipids. ${ }^{\mathbf{9}}$ This is based on the fact that single bonds have a negative diamagnetic anisotropy, while that of multiple bonds is positive. ${ }^{29}$ Adding a biphenyl group along the alkyl chains significantly increases the $\Delta \chi$ of the phospholipids, such that the total anisotropy goes from negative to positive. Consequently, the alignment of the bicelle changes from parallel to perpendicular to the field. The magnetic anisotropy of phospholipid molecules can also be modified by incorporation of certain paramagnetic lanthanide ions. It has been experimentally demonstrated that doping of phospholipids with $\mathrm{Eu}^{3+}, \mathrm{Er}^{3+}, \mathrm{Tm}^{3+}$, and $\mathrm{Yb}^{3+}$ changes the magnetic anisotropy from negative to positive. ${ }^{97}$ Incorporation of certain other paramagnetic lanthanide ions like $\mathrm{Dy}^{3+}$ leads to a more negative anisotropy. ${ }^{\mathbf{9 8 , 9 9}}$

Magnetic deformation has also been shown to occur for nonbiological structures. Spherical sexithiophene nanocapsules were deformed into oblate superspheroids in magnetic fields up to $20 \mathrm{~T}^{\mathbf{1 0 0 , 1 0 1}}$ The deformation was visualized by magnetic birefringence and scanning electron microscope imaging of the deformed capsules trapped in a gel (see Fig. 9). Since the $\Delta \chi$ of sexithiophenes is positive, deformation leads to oblate structures rather than prolate as was the case for liposomes. 


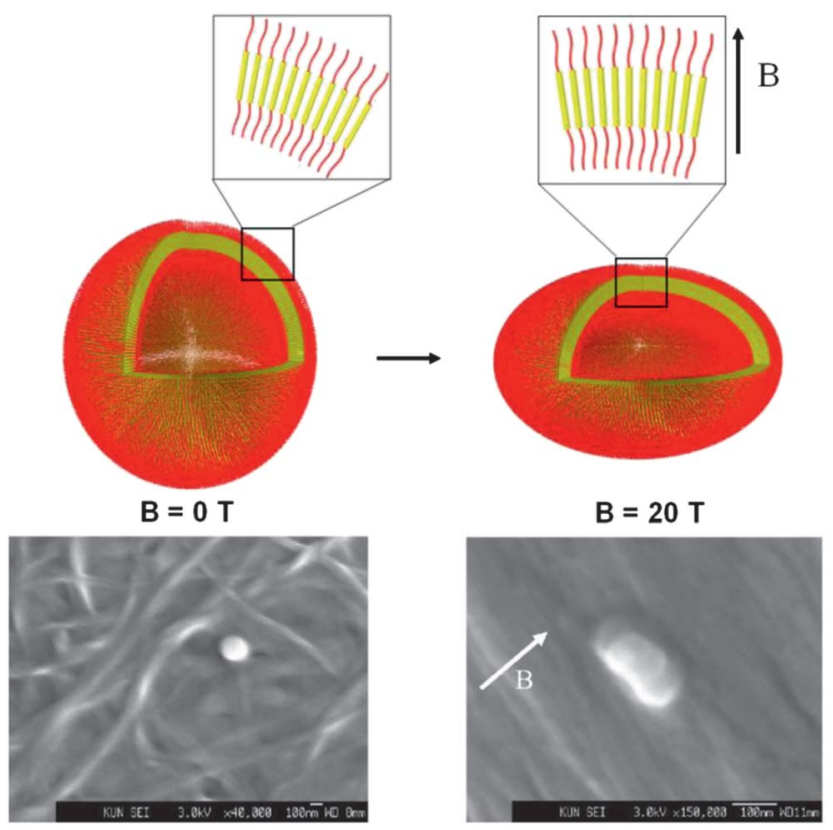

Fig. 9 Left: a sexithiophene nanocapsule stored in a gel at $0 \mathrm{~T}$. Right: a nanocapsule stored in a gel at $20 \mathrm{~T}$. The figure shows how the individual molecules are oriented with respect to the magnetic field Adapted from ref. 100.

Deformation of spherical nano-objects into spheroids could find some interesting applications in the manipulation of their motion. Firstly, a spheroid breaks spherical symmetry and hence the diffusion coefficient decreases along the long axis while it increases along the short axis. ${ }^{102-104}$ Secondly, by tuning the sign of $\Delta \chi$, one can differentiate between a prolate $(\Delta \chi<0)$ and an oblate $(\Delta \chi>0)$ spheroid, where the first is a sphere elongated along one axis while the second is a sphere which is contracted along one axis. This differentiation made it possible to choose the direction of least drag.

In our group, we used diamagnetic amphiphilic block copolymers to create several supramolecular morphologies like micelles, rods, vesicles and so-called stomatocytes. ${ }^{\mathbf{1 0 5 - 1 1 0}}$ The latter architectures were filled with platinum spheres to construct nanomotors. Since the block co-polymers exhibit diamagnetic anisotropy, we expect that high magnetic fields will form an interesting tool to controllably and reversibly modify the conformations and alignment of these structures. Furthermore, chemical modification of the individual block copolymers makes it possible to alter the size and sign of the diamagnetic anisotropy. Magnetic manipulation of diamagnetic supramolecular structures might therefore be an exciting new field of interest.

\subsection{Inhomogeneous magnetic fields and diamagnets}

Magneto-Archimedes levitation was used to separate mixtures of diamagnetic matter ${ }^{31-34}$ or mixtures of diamagnetic and paramagnetic matter. ${ }^{\mathbf{1 1 1}}$ Winkleman et al. showed that polystyrene particles differing in the amount of $\mathrm{CH}_{2} \mathrm{Cl}$ groups could be separated by a pair of permanent magnets when placed inside a paramagnetic solution of gadolinium salts. ${ }^{32}$ Another example is the levitation and separation of diamagnetic sodium chloride and potassium chloride particles inside pressurized paramagnetic gas (oxygen) at magnetic field gradients of $410 \mathrm{~T}^{2}$ $\mathrm{m}^{-1} \cdot{ }^{24}$ Pressurized oxygen has also been used to separate different biological materials like DNA and cholesterol. ${ }^{33,34}$

In the area of microfluidics diamagnetic manipulation can be performed with permanent magnets, since they can be positioned very close to microchannels or they can be integrated in microfluidic chips. ${ }^{112}$ This can create relatively strong magnetic field gradients over small volumes. Zhu et al. used this principle to deflect diamagnetic polystyrene particles in a paramagnetic magnesium chloride solution. ${ }^{111}$ The group of Pamme also experimented with repulsion of diamagnetic microparticles in microfluidic chips. ${ }^{\mathbf{1 1 3 , 1 1 4}}$ Diamagnetic polymer microspheres were placed in paramagnetic manganese chloride solutions and exposed to an inhomogeneous magnetic field created by a superconducting magnet to create a repelling force leading to deflection of the diamagnetic particles.

Another example is the trapping of diamagnetic polystyrene beads and living cells in a paramagnetic buffer between two permanent magnets placed $30 \mu \mathrm{m}$ apart. ${ }^{27}$ The two magnets faced each other with their north poles, creating a large magnetic field gradient. The diamagnetic particles were trapped in the midst between the two magnets where the magnetic field strength was lowest. The authors described this technique as a viable alternative to optical tweezers, being able to trap a wide variety of materials with larger sizes than those held by optical traps.

\subsection{Rotating magnetic fields and diamagnets}

In the previous sections, it was discussed that static magnetic fields can be used to align particles with their axis of highest magnetic susceptibility parallel to the magnetic field. The drawback of this type of alignment is that it leaves the other two axes free to rotate perpendicular to the magnetic field..$^{115}$

Kimura et al. have shown that it is also possible to align diamagnetic particles along their axis of lowest magnetic susceptibility by using a rotating magnetic field. Nylon fibers were used with $\chi_{z}<\chi_{y}=\chi_{x}<0 .{ }^{116}$ In a static magnetic field, the fibers aligned perpendicular to the magnetic field, but were still able to rotate in a plane perpendicular to the field. The authors showed, both experimentally and theoretically, that the use of a rotating magnetic field led to alignment of the fibers along the molecular $z$-axis. This was achieved by first aligning the fibers by a static magnetic field, followed by exposure to a rotating magnetic field. An alternative method used alternating exposure to a homogeneous magnetic field in two perpendicular directions (see Fig. 10). Although the fiber dimensions were on the order of millimetres, the authors calculated that this procedure would also work for smaller particles (dimensions on the order of tenths of nanometers) if magnetic fields of $10 \mathrm{~T}$ or more are used. Rotating magnetic fields have also been used to align chiral cellulosic microfibers that form a nematic liquid crystal phase uniaxially which resulted in the unwinding of the helical structure. ${ }^{\mathbf{1 1 7}}$ Theoretical research has also predicted that 
1. No magnetic field

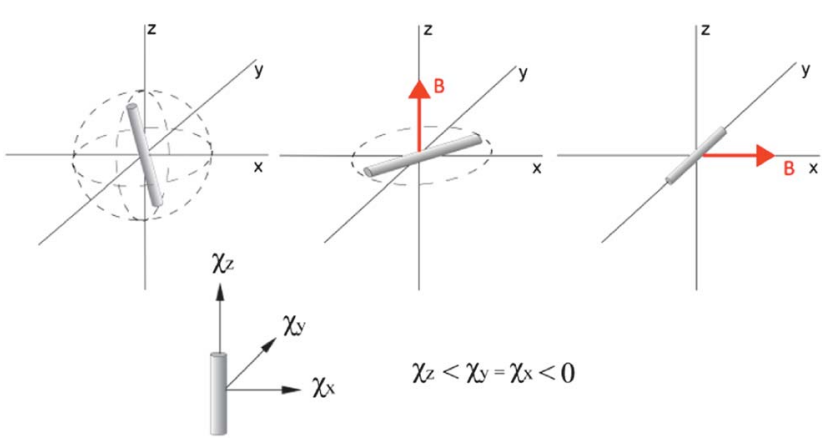

Fig. 10 Alignment of a nylon fiber can be achieved by an alternating magnetic field. When no field is present the fiber is free to rotate along all three axes (1). A field along the $z$-axis aligns the fiber on the $x y$ plane, but the fiber is still free to rotate within this plane (2). A subsequent switch of the magnetic field to the $x$-direction will align the fiber in the $x y$-plane along the $y$-axis. Adapted from ref. 116 .

it will be possible to align molecules with a diamagnetic anisotropy of $\chi_{\mathrm{z}}<\chi_{\mathrm{y}}<\chi_{\mathrm{x}}<0$ by a rotating elliptic magnetic field. ${ }^{115}$ These techniques also apply to paramagnetic particles with a similar magnetic anisotropy.

\section{Conclusions}

Most research on the magnetic propulsion and steering of micro- and nanostructures has been performed with ferro- and paramagnetic (nano)particles. The most important reason for this is their relatively strong response to magnetic fields compared to diamagnetic matter. Manipulation of movement was achieved with homogeneous, inhomogeneous, rotating and oscillating magnetic fields.

Exposure of diamagnetic materials to (strong) magnetic fields can be used to align them and/or to induce shape changes that make them anisotropic. This allows for reversible modifications and, therefore, creates new opportunities to control the motion of novel micro- and nanostructures. Diamagnetic manipulation with permanent magnets has already been employed in microfluidics where magnetic repulsion of diamagnetic particles is enhanced by placing them in paramagnetic solutions. Deformation of supramolecular structures built from diamagnetic molecules still requires magnetic fields up to several Tesla, depending on the diamagnetic anisotropy of the molecules used.

Most research, for all magnetic materials, has been performed on microstructures rather than nanostructures. One reason for this is that smaller structures experience smaller magnetic forces, meaning that they are more easily disturbed by Brownian motion. This problem could be addressed by the use of higher magnetic fields. A second reason is the lack of in situ techniques that allow velocity determination and tracking of nanoparticles while exposing them to controllable magnetic fields. Optical microscopes are easily adapted with small bar or electromagnets but their resolution is usually limited to the micron size. Possible techniques that could be developed are in situ confocal microscopy or in situ dynamic light scattering in stronger magnetic fields of $2 \mathrm{~T}$ or more. This could allow for miniaturization of para-, dia- and ferromagnetic nanostructures that can still compete with Brownian motion and whose movements can be tracked.

In summary, we foresee many opportunities in the miniaturization of para-, dia- and ferromagnetic structures using higher magnetic fields combined with the use of new in situ tracking methods that allow the motion of nanostructures to be followed in real-time.

\section{Acknowledgements}

R. S. M. Rikken acknowledges the Graduate School for Molecules and Materials for a PhD position and NWO for its corresponding grant. D. A. Wilson acknowledges funding from the European Research Council under the European Union's Seventh Framework Programme (FP7/2007-2012)/ERC-StG 307679 “StomaMotors". R. J. M. Nolte acknowledges support from the European Research Council (ERC Advanced grant 290886-ALPROS) and from the Ministry of Education, Culture and Science (Gravity program 024.001.035).

\section{Notes and references}

1 D. Kagan, R. Laocharoensuk, M. Zimmerman, C. Clawson, S. Balasubramanian, D. Kang, D. Bishop, S. Sattayasamitsathit, L. Zhang and J. Wang, Small, 2010, 6, 2741.

2 N. Pamme, Lab Chip, 2006, 6, 24.

3 M. A. M. Gijs, F. Lacharme and U. Lehmann, Chem. Rev., 2010, 110, 1518.

4 M. A. M. Gijs, Microfluid. Nanofluid., 2004, 1, 22.

5 N. Xia, T. P. Hunt, B. T. Mayers, E. Alsberg, G. M. Whitesides, R. M. Westervelt and D. E. Ingber, Biomed. Microdevices., 2006, 8, 299.

6 A. A. Solovev, S. Sanchez, M. Pumera, Y. F. Mei and O. G. Schmidt, Adv. Funct. Mater., 2010, 20, 2430.

7 A. A. Shah, H. Kang, K. L. Kohlstedt, K. H. Ahn, S. C. Glotzer, C. W. Monroe and M. J. Solomon, Small, 2012, 8, 1551.

8 P. D. Vogel, Eur. J. Pharm. Biopharm., 2005, 60, 267.

9 K. Svoboda, C. F. Schmidt, B. J. Schnapp and S. M. Block, Nature, 1993, 365, 721.

10 Y. Lu, L. Dong, L.-C. Zhang, Y.-D. Su and S.-H. Yu, Nano Today, 2012, 7, 297-315.

11 B. L. Feringa, J. Org. Chem., 2007, 72, 6635.

12 W. R. Browne and B. L. Feringa, Nat. Nanotechnol., 2006, 1, 25.

13 R. Kapral, J. Chem. Phys., 2013, 138, 020901.

14 J. Wang and K. M. Manesh, Small, 2010, 6, 338.

15 J. Wang and W. Gao, ACS Nano, 2012, 6, 5745.

16 S. J. Ebbens and J. R. Howse, Soft Matter, 2010, 6, 726.

17 P. Fischer and A. Ghosh, Nanoscale, 2011, 3, 557.

18 N.-T. Nguyen, Microfluid. Nanofluid., 2012, 12, 1.

19 G. Friedman and B. Yellen, Curr. Opin. Colloid Interface Sci., 2005, 10, 158. 
20 D. J. Griffiths, Introduction to Electrodynamics, Benmjamin Cummings, 3th edn, 2008, ch. 6.

21 Magneto-Science, ed. M. Yamaguchi and Y. Tanimoto, Kodansha Springer, 1st edn, 2006, ch. 1.

22 C. Kittel, Introduction to Solid State Physics, Wiley, 8th edn, 2005, ch. 11.

23 P. W. Atkins and J. De Paula, Physical Chemistry, Oxford, 8th edn, 2006, ch. 15.

24 G. Küstler, Rev. Roum. Sci. Tech., Ser. Electrotech. Energ., 2007, 52, 265.

25 A. Sakaguchi, A. Hamasaki and S. Ozeki, Chem. Lett., 2012, 41, 342.

26 M. Suwa and H. Watarai, Anal. Chim. Acta, 2011, 690, 137.

27 A. Winkleman, K. L. Gudiksen, D. Ryan and G. M. Whitesides, Appl. Phys. Lett., 2004, 85, 2411.

28 I. F. Lyuksyutov, D. G. Naugle and K. D. D. Rathnayaka, Appl. Phys. Lett., 2004, 85, 1817.

29 G. Maret and K. Dransfeld, Strong and Ultrastrong Magnetic Fields and Their Applications, Springer-Verlag, Berlin, 1985, ch. 4 .

30 A. R. Urbach, J. C. Love, M. G. Prentiss and G. M. Whitesides, J. Am. Chem. Soc., 2003, 125, 12704.

31 Y. Ikezoe, T. Kaihatsu, S. Sakae, H. Uetake, N. Hirota and K. Kitazawa, Energy Convers. Manage., 2002, 43, 417.

32 A. Winkleman, R. Perez-Castillejos, K. L. Gudiksen, S. T. Phillips, M. Prentiss and G. M. Whitesides, Anal. Chem., 2007, 79, 6542.

33 N. Hirota, M. Kurashige, M. Iwasaka, M. Ikehata, H. Uetake, T. Takayama, H. Nakamura, Y. Ikezoe, S. Ueno and K. Kitazawa, Physica B, 2004, 346, 267.

34 K. Yokoyama, N. Hirota and M. Iwasaka, IEEE Trans. Appl. Supercond., 2007, 17, 2181.

35 M. V. Berry and A. K. Geim, Eur. J. Phys., 1997, 18, 307.

36 E. Beaugnon and R. Tournier, Nature, 1991, 349, 470.

37 M. D. Simon and A. K. Geim, J. Appl. Phys., 2000, 87, 6200.

38 K. A. Mirica, S. T. Phillips, C. R. Mace and G. M. Whitesides, J. Agric. Food Chem., 2010, 58, 6565.

39 S. S. Shevkoplyas, A. C. Siegel, R. M. Westervelt, M. G. Prentiss and G. M. Whitesides, Lab Chip, 2007, 7, 1294.

40 T. R. Kline, W. F. Paxton, T. E. Mallouk and A. Sen, Angew. Chem., Int. Ed., 2005, 44, 744.

41 R. F. Ismagilov, A. Schwartz, N. Bowden and G. M. Whitesides, Angew. Chem., Int. Ed., 2002, 41, 652.

42 L. Baraban, D. Makarov, R. Streubel, I. Mönch, D. Grimm, S. Sanchez and O. G. Schmidt, ACS Nano, 2012, 6, 3383.

43 K. K. Dey, D. Sharma, S. Basu and A. Chattopadhyay, J. Chem. Phys., 2008, 129, 121101.

44 K. K. Dey, K. K. Senapati, P. Phukan, S. Basu and A. Chattopadhyay, J. Phys. Chem. C, 2011, 115, 12708.

45 P. K. Challa, O. Curtiss, J. C. Williams, R. Twieg, J. Toth, S. McGill, A. Jákli, J. T. Gleeson and S. N. Sprunt, Phys. Rev. E: Stat., Nonlinear, Soft Matter Phys., 2012, 86, 011708. 46 N. Hirota and T. Ode, Rev. Sci. Instrum., 2006, 77, 036107. 47 S. Foner, IEEE Trans. Appl. Supercond., 1995, 5, 121.

48 J.-B. Mathieu, S. Martel, L. Yahia, G. Soulez and G. Beaudoin, Biomed. Mater. Eng., 2005, 15, 367.
49 J.-P. Mathieu, G. Beaudoin and S. Martel, IEEE Trans. Biomed. Eng., 2006, 53, 292.

50 J.-P. Mathieu and S. Martel, Conf. Proc. IEEE Eng. Med. Biol. Soc., 2006, 1, 472.

51 Q. A. Pankhurst, J. Connolly, S. K. Jones and J. Dobson, J. Phys. D: Appl. Phys., 2003, 36, R167.

52 Q. A. Pankhurst, N. T. K. Thanh, S. K. Jones and J. Dobson, J. Phys. D: Appl. Phys., 2009, 42, 224001.

53 T. Honda, K. I. Arai and K. Ishiyama, IEEE Trans. Magn., 1996, 32, 5085.

54 L. Zhang, J. J. Abbott, L. Dong, B. E. Kratochvil, D. Bell and B. J. Nelson, Appl. Phys. Lett., 2009, 94, 064107.

55 U. K. Cheang, D. Roy, J. H. Lee and M. J. Kim, Appl. Phys. Lett., 2010, 97, 213704.

56 P. Garstecki, P. Tierno, D. B. Weibel, F. Sagués and G. M. Whitesides, J. Phys.: Condens. Matter, 2009, 21, 204110.

57 P. Tierno, R. Golestanian, I. Pagonabarraga and F. Sagués, J. Phys. Chem. B, 2008, 112, 16525.

58 W. Gao, K. M. Manesh, J. Hua, S. Sattayasamitsathit and J. Wang, Small, 2011, 7, 2047.

59 P. Dhar, C. D. Swayne, T. M. Fisher, T. Kline and A. Sen, Nano Lett., 2007, 7, 1010.

60 A. Jordan, R. Scholz, P. Wust, H. Fähling and R. Felix, J. Magn. Magn. Mater., 1999, 201, 413.

61 C. S. Kumar and F. Mohammad, Adv. Drug Delivery Rev., 2011, 63, 789.

62 B. Mehdaoui, J. Carrey, M. Stadler, A. Cornejo, C. Nayral, F. Delpech, B. Chaudret and M. Respaud, Appl. Phys. Lett., 2012, 100, 052403.

63 L. Baraban, R. Streubel, D. Makarov, L. Han, D. Karnaushenko, O. G. Schmidt and G. Cuniberti, ACS Nano, 2013, 7, 1360.

64 A. Butykai, A. Orbán, V. Kocsis, D. Szaller, S. Bordács, E. Tátrai-Szekeres, L. F. Kiss, A. Bóta, B. G. Vértessy,

T. Zelles and I. Kézsmárki, Sci. Rep., 2013, 3, 1431.

65 P. Tierno, R. Albalat and F. Sagués, Small, 2010, 6, 1749.

66 N. Chaturvedi, Y. Hong, A. Sen and D. Velegol, Langmuir, 2010, 26, 6308.

67 L. Baraban, D. Makarov, O. Schmidt, G. Cuniberti, P. Leiderer and A. Erbe, Nanoscale, 2013, 5, 1332.

68 M. Fujiwara, D. Kodoi, W. Duan and Y. Tanimoto, J. Phys. Chem. B, 2001, 105, 3343.

69 K. Chie, M. Fujiwara, Y. Fujiwara and Y. Tanimoto, J. Phys. Chem. B, 2003, 107, 14374.

70 M. Fujiwara, K. Chie, J. Sawai, D. Shimizu and Y. Tanimoto, J. Phys. Chem. B, 2004, 108, 3531.

71 M. Fujiwara, K. Mitsuda and Y. Tanimoto, J. Phys. Chem. B, 2006, 110, 13965.

72 O. Güell, F. Sagués and P. Tierno, Adv. Mater., 2011, 23, 3674.

73 P. Dhar, Y. Cao, T. Kline, P. Pal, C. Swayne, T. M. Fischer, B. Miller, T. E. Mallouk, A. Sen and T. H. Johansen, J. Phys. Chem. C, 2007, 111, 3607.

74 P. Tierno, F. Sagués, T. H. Johansen and T. M. Fischer, Phys. Chem. Chem. Phys., 2009, 11, 9615.

75 E. M. Furst, C. Suzuki, M. Fermigier and A. P. Gast, Langmuir, 1998, 14, 7334. 
76 A. K. Vuppu, A. A. Garcia and M. A. Hayes, Langmuir, 2003, 19, 8646.

77 S. Krishnamurthy, A. Yadav, P. E. Phelen, R. Calhoun, A. K. Vuppu, A. A. Garcia and M. A. Hayes, Microfluid. Nanofluid., 2008, 5, 33.

78 S. L. Biswal and A. P. Gast, Phys. Rev. E: Stat., Nonlinear, Soft Matter Phys., 2004, 69, 041406.

79 S. L. Biswal and A. P. Gast, Anal. Chem., 2004, 76, 6448.

80 M. Karle, J. Miwa, G. Czilwik, V. Auwärter, G. Roth, R. Zengerle and F. Von Stetten, Lab Chip, 2010, 10, 3284.

81 M. Karle, J. Wöhrle, J. Miwa, N. Paust, G. Roth, R. Zengerle and F. Von Stetten, Microfluid. Nanofluid., 2011, 10, 935.

82 R. Dreyfus, J. Baudry, M. L. Roper, M. Fermigier, H. A. Stone and J. Bibette, Nature, 2005, 437, 862.

83 Y. Nagaoka, H. Morimoto and T. Maekawa, Langmuir, 2011, 27, 9160.

84 P. C. M. Christianen, I. O. Shklyarevskiy, M. I. Boamfa and J. C. Maan, Physica B, 2004, 346-347, 255.

85 I. O. Shklyarevskiy, M. I. Boamfa, P. C. M. Christianen, F. Touhari, H. van Kempen, G. Deroover, P. Callant and J. C. Maan, J. Chem. Phys., 2002, 116, 8407.

86 I. O. Shklyarevskiy, P. Jonkheijm, P. C. M. Christianen, A. P. H. J. Schenning, A. Del Guerzo, J.-P. Desvergne, E. W. Meijer and J. C. Maan, Langmuir, 2005, 21, 2108.

87 I. O. Shklyarevskiy, P. Jonkheijm, N. Stutzmann, D. Wasserberg, H. J. Wondergem, P. C. M. Christianen, A. P. H. J. Schenning, D. M. de Leeuw, Z. Tomović, J. Wu, K. Müllen and J. C. Maan, J. Am. Chem. Soc., 2005, 127, 16233.

88 J. C. Gielen, M. Wolffs, G. Portale, W. Bras, O. Henze, A. F. M. Kilbinger, W. J Feast, J. C. Maan, A. P. H. J. Schenning and P. C. M. Christianen, Langmuir, 2009, 25, 1272.

89 K. Guevorkian and J. M. Valles Jr, Biophys. J., 2006, 90, 3004.

90 W. Helfrich, Phys. Lett., 1973, 43A, 409.

91 W. Helfrich, Z. Naturforsch., C: J. Biosci., 1973, 28, 693.

92 T. S. Tenforde and R. P. Liburdy, J. Theor. Biol., 1988, 133, 385.

93 S. Ozeki, H. Kurashima and H. Abe, J. Phys. Chem. B, 2000, 104, 5657.

94 X. Qiu, P. A. Mirau and C. Pidgeon, Biochim. Biophys Act, 1993, 1147, 59.

95 M. A. Kiselev, M. Janich, P. Lesieur, A. Hoell, J. Oberdisse, G. Pepy, A. M. Kisselev, I. V. Gapienko, T. Gutberlet and V. L. Aksenov, Appl. Phys. A, 2002, 74, 1239.

96 C. Tan, B. M. Fung and G. Cho, J. Am. Chem. Soc., 2002, 124, 11827.
97 R. S. Prosser, S. A. Hunt, J. A. DiNatale and R. R. Vold, J. Am. Chem. Soc., 1996, 118, 269.

98 P. Beck, M. Liebi, J. Kohlbrecher, T. Ishikawa, H. Rüegger, H. Zepik, P. Fischer, P. Walde and E. Windhab, J. Phys. Chem. B, 2010, 114, 174.

99 M. Liebi, P. G. van Rhee, P. C. M. Christianen, J. Kohlbrecher, P. Fischer, P. Walde and E. J. Windhab, Langmuir, 2013, 29, 3467.

100 I. O. Shklyarevski, P. Jonkheijm, P. C. M. Christianen, A. P. H. J. Schenning, E. W. Meijer, O. Henze, A. F. M. Kilbinger, W. J. Feast, A. Del Guerzo, J.-P. Desvergne and J. C. Maan, J. Am. Chem. Soc., 2005, 127, 1112.

101 O. V. Manyuhina, I. O. Shklyarevski, P. Jonkheim, P. C. M. Christianen, A. Fasolino, M. I. Katnelson, A. P. H. J. Schenning, E. W. Meijer, O. Henze, A. F. M. Kilbinger, W. J. Feast and J. C. Maan, Phys. Rev. Lett., 2007, 98, 146101.

102 F. Perrin, Le Journal de Physique et le Radium, 1934, 7, 497. 103 H. Shimiuzu, J. Chem. Phys., 1962, 37, 765.

104 R. Vasanthi, S. Bhattacharyya and B. Bagchi, J. Chem. Phys., 2002, 116, 1092.

105 J. A. Opsteen, J. J. L. M. Cornelissen and J. C. M. van Hest, Pure Appl. Chem., 2004, 76, 1309.

106 M. G. Jeong, J. C. M. van Hest and K. T. Kim, Chem. Commun., 2012, 48, 359069.

107 K. T. Kim, J. Zhu, S. A. Meeuwissen, J. J. L. M. Cornelissen, D. J. Pochan, R. J. M. Nolte and J. C. M. van Hest, J. Am. Chem. Soc., 2010, 132, 12522.

108 S. A. Meeuwissen, K. Kim, Y. Chen, D. J. Pochan and J. C. M. van Hest, Angew. Chem., Int. Ed., 2011, 50, 7070.

109 D. A. Wilson, R. J. M. Nolte and J. C. M. van Hest, Nat. Chem., 2012, 4, 268.

110 D. A. Wilson, R. J. M. Nolte and J. C. M. van Hest, J. Am. Chem. Soc., 2012, 134, 9894.

111 F. Mishima, T. Terada, Y. Akiyama and S. Nishijima, IEEE Trans. Appl. Supercond., 2011, 21, 2059.

112 J. Zhu, L. Liang and X. Xuan, Microfluid. Nanofluid., 2012, 12,65 .

113 M. D. Tarn, N. Hirota, A. Iles and N. Pamme, Sci. Technol. Adv. Mater., 2009, 10, 014611.

114 M. Vojtíšek, M. D. Tarn, N. Hirota and N. Pamme, Microfluid. Nanofluid., 2012, 13, 625.

115 T. Kimura and M. Yoshino, Langmuir, 2005, 21, 4805.

116 T. Kimura, M. Yoshino, T. Yamane, M. Yamato and M. Tobita, Langmuir, 2004, 20, 5669.

117 F. Kimura, T. Kimura, M. Tamura, A. Hirai, M. Ikuno and F. Horii, Langmuir, 2005, 21, 2034. 